\title{
Influence of Micronized Chitosan on Antioxidative Activities in Grape Juice
}

\author{
Po-Jung Chien ${ }^{1 *}$, Chi-Ming Li ${ }^{2}$, Chi-Heng Lee ${ }^{1}$, Hsuan-Hung Chen ${ }^{1}$ \\ ${ }^{1}$ Department of Horticulture and Biotechnology, Chinese Culture University, Taipei, Taiwan; ${ }^{2}$ Graduate Institute of Biotechnology, \\ Chinese Culture University, Taipei, Taiwan. \\ Email: "pojungchien@gmail.com
}

Received June $5^{\text {th }}, 2013$; revised July $5^{\text {th }}, 2013$; accepted July $12^{\text {th }}, 2013$

Copyright (C) 2013 Po-Jung Chien et al. This is an open access article distributed under the Creative Commons Attribution License, which permits unrestricted use, distribution, and reproduction in any medium, provided the original work is properly cited.

\begin{abstract}
The antioxidant activity of chitosan before micronization (BMC, average particle size of $1850 \pm 26.3 \mu \mathrm{m}$ ) and after micronization (AMC, average particle size of $1.37 \pm 0.2 \mu \mathrm{m}$ ) in grape juice was studied. Antioxidant activity was determined, including that of DPPH radicals, hydrogen peroxide and superoxide anion radicals, as well as ABTS radicals of $\mathrm{BMC}$ or $\mathrm{AMC}$ in grape juice. AMC exhibits stronger scavenging activity toward DPPH radicals, superoxide anion radicals and hydrogen peroxide than BMC. At a concentration of $1.0 \mathrm{mg} / \mathrm{mL}$, AMC in grape juice exhibited $90.0 \%$, $97.3 \%$ and $88.7 \%$ scavenging activities toward DPPH radicals, hydrogen peroxide and superoxide anion radicals, respectively. The TEAC (Trolox Equivalent Antioxidant Capacity) values of AMC $(3.94 \pm 0.19)$ greatly exceeded those of BMC $(2.21 \pm 0.10)$ in grape juice. The in vitro results in this investigation suggest the possibility that AMC can increase the antioxidant activity in grape juice. However, comprehensive studies must be performed to ascertain the in vivo safety of AMC in experimental animal models.
\end{abstract}

Keywords: Micronization; Chitosan; Grape Juice; Antioxidative Activity

\section{Introduction}

Chitosan ( $\beta$-(1-4)-2-amino-2-deoxy-D-glucose) is a linear hydrophilic polysaccharide polymer of d-glucosamine. It is abundant in nature and is present in the exoskeleton of crustaceans, including crabs and shrimp [1]. Chitosan, being a cationic polysaccharide under neutral or basic $\mathrm{pH}$ conditions, contains free amino groups and is therefore insoluble in water. At acidic $\mathrm{pH}$ values, amino groups undergo protonation thus, making chitosan soluble in water. It breaks down slowly into harmless products (amino sugars), which are entirely absorbed by the human body [2].

In recent years, various researchers have evaluated the antioxidant activity of chitosan derivatives. For example, Xie et al. [3] revealed that hexanolychitin and $N$-benzoylhexanoyl chitosan can trap peroxide radicals in an organic solvent when 2,2'-azobis (2,4-dimethylvaleronitrile) initiates the radical chain reaction. Lin and Chou [4] demonstrated that disaccharide chitosan derivatives exhibit a range of antioxidative activities. Esumi et al. [5] showed that gold-chitosan nanocomposites can suppress

${ }^{*}$ Corresponding author. the activity of hydroxyl radicals. Xing et al. [6] determined the effects of molecular weight and/or the degree of substitution of sulfated polysaccharides on their antioxidant activity. The health advantages of consuming grape juice, including improved endothelial function, increased antioxidant capacity of serum, protection of LDLs against oxidation, reduced native plasma protein oxidation, and reduced platelet aggregation, have been reported upon [7]. However, the effect of micronized chitosan on the antioxidant activity of grape juice has not yet been reported upon. This study compares the effects of micronized chitosan on the antioxidant activity, and on the possible antioxidant effects of grape juice. This investigation will evaluate the antioxidant capacity of BMC (or AMC) in grape juice. Antioxidant activities were evaluated using various in vitro assay systems, involving, for example, DPPH, superoxide, hydroxyl radicals and ABTS.

\section{Materials and Methods}

\subsection{Micronization of Chitosan and Chemicals}

Crab-shell chitosan with $86.70 \%$ N-deacetylation as a 
powder were obtained from VA \& G Bioscience Inc (Taoyuan, Taiwan) and micronzied using a high-speed planetary ball-mill (PM100, Retsch, Germany), by the approach of that was proposed by Chau et al. [8]. In the ball-milling process, sample and agate balls with a diameter of $3 \mathrm{~mm}$ in a volume ratio of 1:1 ( $\sim 165 \mathrm{ml}$ each) were added to an agate grinding bowl with a volume of $500 \mathrm{ml}$. The sample was then ground for $2 \mathrm{~h}$ using agate balls. Before and after the chitosan samples were estimated using a laser particle size analyzer (Analysette 22-Economy, Fritsch, Germany). All other chemicals were obtained commercially and were of analytical grade. Nitro blue tetrazolium (NBT), phenazine methosulfate (PMS), hydrogen peroxide $\left(\mathrm{H}_{2} \mathrm{O}_{2}\right)$, thiobarbituric acid (TBA), ethylene diamine tetra-acetic acid (EDTA), ferrozine, nicotinamide adenine dinucleotide-reduced (NADH), trichloroacetic acid (TCA), potassium ferricyanide and ferric chloride were obtained from Sigma Chemicals Co., St. Louis, MO, USA.

The antioxidant activities of micronized individual chitosans were evaluated in grape juice.

\subsection{Preparing Chitosan Solution}

In the preparation of $1.0 \mathrm{~L}$ of $0.1 \%-1.0 \%$ chitosan solutions, $0.1,0.5$ and $1.0 \mathrm{~g}$ of chitosan were dispersed in 900 $\mathrm{ml}$ of distilled water, to which $50 \mathrm{ml}$ of glacial acetic acid was added to dissolve the chitosan. The $\mathrm{pH}$ of each solution was adjusted to 5.0 by adding $0.1 \mathrm{M} \mathrm{NaOH}$ and each solution was made up to $1.0 \mathrm{~L}$. An acid solution without chitosan at pH 5.0 was used as a control.

\subsection{Preparing Grape Juice That Contains Micronized Chitosans}

Clear, UTH-treated, shelf-stable grape juice that contained no added preservatives and was packed in laminated, was purchased from a local retailer. To $45 \mathrm{~mL}$ of this grape juice in a $250 \mathrm{~mL}$ Erlenmeyer flask was added $5 \mathrm{~mL}$ of micronized chitosan solution. To the control flask was added $5 \mathrm{~mL}$ of water, rather than chitosan solution. The used chitosan concentrations ranged from 0.1 to $1.0 \mathrm{~g} / \mathrm{L}$ of juice.

\subsection{Scavenging of DPPH Radical}

The effect of chitosans on DPPH radicals was examined using the modified method of Shimada et al. [9]. Briefly, $100 \mu \mathrm{M}$ DPPH solution in methanol was prepared and $1.0 \mathrm{~mL}$ of this solution was added to $4.0 \mathrm{~mL}$ test samples at (various concentrations. The reaction mixture was shaken thoroughly and incubated for $30 \mathrm{~min}$ at room temperature and the absorbance of the resulting solution was determined at $517 \mathrm{~nm}$ against a blank. The percentage inhibition of DPPH was calculated using the following equation:

$$
\begin{aligned}
& \text { Scavenging effect }(\%) \\
& =[1-\text { absorbancesample } / \text { absorbancecontrol }] \times 10
\end{aligned}
$$

\subsection{Hydrogen Peroxide Scavenging Assay}

The activity of chitosan in scavenging hydrogen peroxide was measured by a modified version of the method that was proposed by Yen and Chang [10]. Briefly, $1 \mathrm{~mL}$ of sample was firstly mixed with $400 \mu \mathrm{L}$ of $4 \mathrm{mM} \mathrm{H}_{2} \mathrm{O}_{2}$ solution and incubated for $20 \mathrm{~min}$ at room temperature. It was then supplemented with $600 \mu \mathrm{l}$ of horseradish peroxidase - phenol red solution (HPRase $300 \mu \mathrm{g} / \mathrm{mL}$ and phenol red $4.5 \mathrm{mM}$ in $100 \mathrm{mM}$ phosphate buffer). HPRase was produced by and obtained from Sigma Chemical Co, St Louis, MO, USA. After another 10 min of incubation and $10 \mathrm{~min}$ on ice to terminate the reaction, the absorbance of the sample at $610 \mathrm{~nm}$ was monitored using an automated microplate reader. The scavenging effect was then calculated using the equation,

$$
\begin{aligned}
& \text { Scavenging effect }(\%) \\
& =\left[1-\text { absorbance }_{\text {sample }} / \text { absorbance }_{\text {control }}\right] \times 100
\end{aligned}
$$

\subsection{Superoxide Anion Radical Scavenging Assay}

The superoxide scavenging capacity of chitosans was assayed using the method of Robak and Gryglewski [11]. The reaction mixture contained chitosans at concentration of $0.1-10 \mathrm{mg} / \mathrm{mL}$. First, the reagents were all prepared in $100 \mathrm{mM}$ phosphate buffer (pH 7.4). The reaction mixture contained $50 \mu \mathrm{L}$ of the test sample, $50 \mu \mathrm{L}$ of 300 $\mu \mathrm{M}$ nitrobluetetrazolium (Sigma), $50 \mu \mathrm{L}$ of $936 \mu \mathrm{M}$ $\mathrm{NADH}$ and $50 \mu \mathrm{L}$ of $120 \mu \mathrm{M}$ phenazine methosulfate (Sigma). It was incubated at room temperature for $5 \mathrm{~min}$ and the absorbance was then read at $560 \mathrm{~nm}$ against a blank. The capacity of chitosan to scavenge superoxide radicals was given by the following equation.

$$
\begin{aligned}
& \text { Scavenging effect }(\%) \\
& =\left[1-\text { absorbance }_{\text {sample }} / \text { absorbance }_{\text {control }}\right] \times 100
\end{aligned}
$$

\subsection{ABTS Assay}

Total antioxidant capacity was evaluated using the ABTS modified assay [12-14]. In the most recent version of the trolox equivalent antioxidant capacity (TEAC) assay, an antioxidant is added to a pre-formed ABTS radical solution and, after a fixed period, the remaining $\mathrm{ABTS}^{\bullet+}$ is quantified spectrophotometrically [15]. The reference compound in the TEAC assay is Trolox. The reduction in ABTS $^{\bullet+}$ concentration, which is caused by a particular concentration of antioxidant, is related to reduction of Trolox concentration, and yields the TEAC value of that 
antioxidant [13].

$\mathrm{ABTS}^{\bullet+}$ was formed by reacting $2.45 \mathrm{mM} \mathrm{ABTS}$ salt, of potassium persulfate $\left(\mathrm{K}_{2} \mathrm{~S}_{2} \mathrm{O}_{8}\right)$, was reacted with 7 $\mathrm{mM}$ ABTS salt in $0.01 \mathrm{M}$ phosphate-buffered saline, $\mathrm{pH} 7.4$, for $15 \mathrm{~h}$ at room temperature in the dark. The resulting $\mathrm{ABTS}^{\bullet+}$ radical cations were diluted with 0.01 $\mathrm{M}$ phosphate-buffered saline at $\mathrm{pH} 7.4$ to yield an absorbance of approximately 0.70 at $734 \mathrm{~nm}$. The standard or sample was diluted by a factor of 100 using the ABTS ${ }^{\bullet+}$ solution to a total volume of $1 \mathrm{~mL}$ and allowed to react for $6 \mathrm{~min}$. Absorbance was measured spectrophotometrically at various times. A blank (without a standard or sample) was used as a control and $990 \mu \mathrm{L}$ of PBS was added to the control samples instead. The absorption peak of ABTS $^{\bullet+}$ was at $734 \mathrm{~nm}$. The addition of antioxidant reduced $\mathrm{ABTS}^{\bullet+}$ to its colorless form. The extent of decolorization as a percentage of inhibition of $\mathrm{ABTS}^{\bullet+}$ is determined as a function of concentration and calculated relative to the reactivity of Trolox, a water-soluble ana$\log$ of vitamin E ( $\alpha$-tocopherol).

\subsection{Statistical Analysis}

In this investigation, three analyses of each sample were performed and each experiment was conducted in triplicate $(n=3)$. The mean value and its standard deviation were calculated from the obtained data. These data were then compared with each other using Duncan's multiple range method.

\section{Results and Discussion}

\subsection{Scavenging of DPPH Radicals by BMC or AMC}

The DPPH, a compound with a proton free radical and a characteristic absorption at $517 \mathrm{~nm}$, is decreased greatly upon exposure to proton radical scavengers [16]. In this study, DPPH was utilized to determine the proton scavenging activity of chitosans of BMC or AMC.

Figure 1 plots the DPPH scavenging potentials of $\mathrm{BMC}$ and AMC in grape juice. In the DPPH test, the grape juice reduced the DPPH radicals, exhibiting $21 \%$ scavenging activity. Here, AMC in grape juice exhibited excellent scavenging activity toward DPPH radical. This fact is attributable to the fact that it has a greater hydrogen-donating capacity than does grape juice (control), BMC or AMC. At a concentration of $1.0 \mathrm{mg} / \mathrm{mL}$, the AMC in grape juice scavenged $90 \%$ of DPPH radicals. The data herein concerning the DPPH scavenging potential of BMC in grape juice demonstrate that AMC probably contributed greatly to the observed antioxidant effect.

\subsection{Scavenging Activity of BMC or AMC toward Superoxide Anion Radical}

Superoxides are radicals whose unpaired electrons are on oxygen. While they are relatively weak oxidants, superoxides exhibit limited chemical reactivity, but can form more dangerous species, including singlet oxygen and hydroxyl radicals, which cause the peroxidation of lipids [17].

Figure 2 shows the effect of BMC or AMC on the superoxide anion radical scavenging activities of grape juice. The data show that AMC had stronger scavenging activity toward superoxide anion radicals at concentrations of $1.0 \mathrm{mg} / \mathrm{mL}$ than at $0.1 \mathrm{mg} / \mathrm{mL}(\mathrm{p}<0.05)$. However, AMC had an excellent capacity to scavenge superoxide anion radicals in grape juice. It had the highest scavenging activity in the elimination of superoxide anion radicals $(p<0.05)$ of any of the tested compounds. At a concentration of $1.0 \mathrm{mg} / \mathrm{mL}$, the AMC in grape

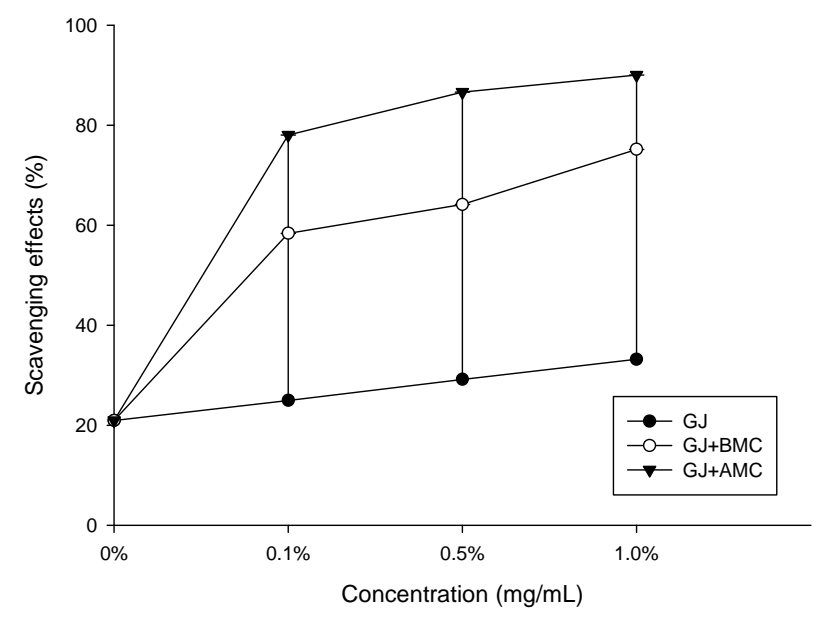

Figure 1. Scavenging effects of BMC or AMC (chitosans) in grape juice on DPPH radicals. Each value is presented as mean \pm SD (n = 3). Grape juice, O; Grape juice + BMC, $\bigcirc$; Grape juice + AMC, $\nabla$.

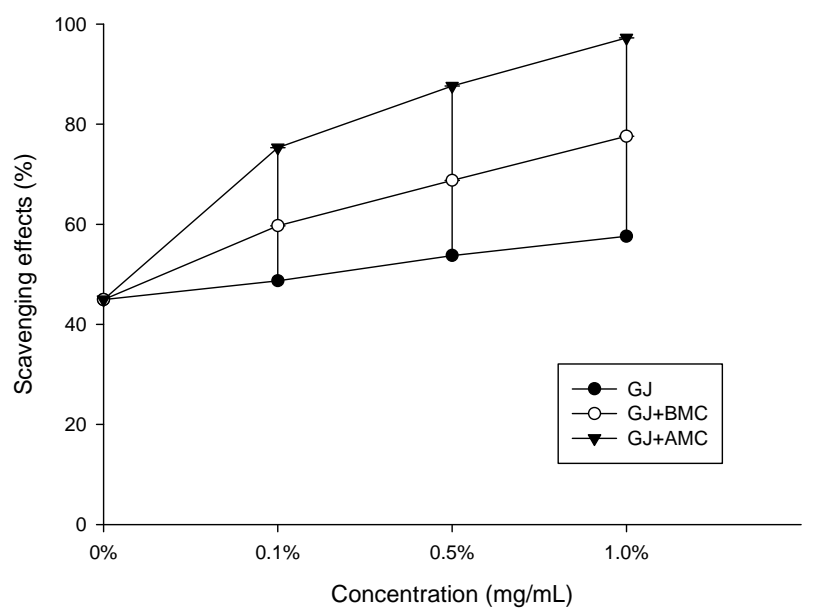

Figure 2. Scavenging effects of BMC or AMC in grape juice on hydrogen peroxide. Each value is presented as mean \pm SD $(n=3)$. Grape juice, O; Grape juice + BMC, $\bigcirc$; Grape juice + AMC, $\nabla$. 
juice scavenged $97.3 \%$ of superoxide anion radicals. They may be more soluble than BMCs. Moreover, superoxides are also known to initiate indirectly lipid peroxidation as a result of the formation of $\mathrm{H}_{2} \mathrm{O}_{2}$, forming precursors to hydroxyl radicals [18]. These findings clearly reveal that the antioxidant activity of AMC increased the capacity of grape juice to scavenge superoxide anion radicals.

\subsection{Hydrogen Peroxide-Scavenging Activity of BMC or AMC}

Some biological systems can generate hydrogen peroxide. High concentrations of hydrogen peroxide can attack various cellular energy-producing systems; as an example of its detrimental effects, it deactivates the glycolytic enzyme glyceraldehydes-3-phosphate dehydrogenase [18]. As a non-radical oxygen-containing reactive agent, it can form the hydroxyl radical, which is the most highly reactive known oxygen radical, in the presence of transition metal ions and thereby participate in a free-radical reaction [19].

Figure 3 shows the hydrogen peroxide scavenging activities of BMC or AMC in grape juice. In the hydrogen peroxide test, the grape juice reduced hydrogen peroxide with a scavenging activity of $25 \%$. In this work, AMC increases the $\mathrm{H}_{2} \mathrm{O}_{2}$-scavenging activity of grape juice. Moreover, the scavenging rate of the chitosans other than $\mathrm{BMC}$ increased with concentration. At a concentration of $1.0 \mathrm{mg} / \mathrm{mL}$, the AMC in grape juice scavenged $88.7 \%$ of hydrogen peroxide, revealing that the tested $\mathrm{BMC}$ and AMC also exhibit considerable $\mathrm{H}_{2} \mathrm{O}_{2}$-scavenging activity in grape juice. AMC was more effective in scavenging $\mathrm{H}_{2} \mathrm{O}_{2}$ in grape juice than was grape juice alone (control) or $\mathrm{BMC}$ in grape juice $(\mathrm{p}<0.05)$. The chealting effects

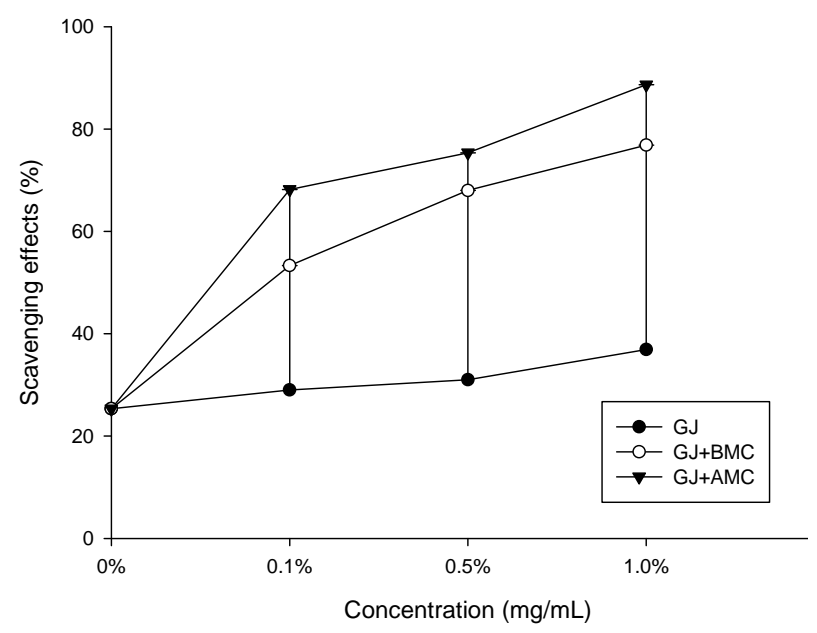

Figure 3. Scavenging effects of BMC or AMC in grape juice on superoxide anion radicals. Each value is presented as mean \pm SD $(n=3)$. Grape juice, $O$; Grape juice + BMC, $\bigcirc$; Grape juice + AMC, $\nabla$.
Table 1. The Trolox equivalent antioxidant capacity (TEAC) value of BMC, AMC in grape juices.

\begin{tabular}{cc}
\hline Compounds & TEAC Persulfate Decolorization Assay ${ }^{\mathrm{a}}$ \\
\hline Trolox & $1.00 \pm 0.01$ \\
BMC & $0.76 \pm 0.02$ \\
AMC & $1.89 \pm 0.07$ \\
GJ & $1.05 \pm 0.04$ \\
GJ + BMC & $2.21 \pm 0.10$ \\
GJ + AMC & $3.94 \pm 0.19$ \\
\hline
\end{tabular}

${ }^{a}$ Applying the $\mathrm{ABTS}^{\bullet+}$ decolorization assay (based on potassium persulfate), the value derived from the area under the time-dependency curve. ${ }^{\mathrm{b}} \mathrm{GJ}$ : grape juice; GJ + BMC: grape juice with before micronization chitosan; GJ + AMC: grape juice with after micronization chitosan. ${ }^{c}$ Data are presented as mean \pm SD. Experiments were performed three times $(n=3)$.

of AMC on metal ions may be responsible for its inhibittion of deoxyribose oxidation.

\subsection{Effect of Trolox Equivalent Antioxidant Capacity (TEAC) on BMC or AMC}

Table 1 presents the effect of BMC or AMC on antioxidant capacity, expressed as TEAC. The behaviors of BMC and $\mathrm{AMC}$ alone differ from those in grape juice. BMC reduced the TEAC value of grape juice to $0.76 \pm 0.02$. However, AMC increased the TEAC value of grape juice to $3.94 \pm 0.19$. The TEAC values of the flavanones, obtained from radical DPPH, were lower than those obtained from the radical ABTS [20]. A possible cause of the considerable rise in TEAC of grape juice is the increase in antioxidant potential polyphenols in the intermediate stages of oxidation. However, further investigations must be performed to elucidate the antioxidant mechanisms of AMC in grape juice.

\section{Conclusion}

The results of this work indicate that AMC (chitosan) exhibit antioxidant activity and free radical scavenging activity, including toward DPPH radicals, hydrogen peroxide and superoxide anion radicals. The assays herein were useful in determining the antioxidant capacities of AMC, which have important applications in the food industry. This study showed that AMC can increase the antioxidant activity of grape juice. However, in vivo antioxidant activity and its various antioxidant mechanisms must be examined further.

\section{Acknowledgements}

The authors would like to thank the National Science Council of the Republic of China, Taiwan (Contract No. NSC96-2313-B-034-006-MY3) and VA\&G Bioscience 
Inc. for financially supporting this research.

\section{REFERENCES}

[1] M. Fukuda, N. A. Peppas and J. W. McGinity, "Properties of Sustained Release Hot-Melt Extruded Tablets Containing Chitosan and Xanthan Gum," International Journal of Pharmaceutics, Vol. 310, No. 1-2, 2006, pp. 90100. doi:10.1016/j.ijpharm.2005.11.042

[2] S. A. Agnihotri, N. N. Mallikarjuna and T. M. Aminabhavi, "Recent Advances on Chitosan-Based Micro- and Nanoparticles in Drug Delivery," Journal of Controlled Release, Vol. 100, No. 1, 2004, pp. 5-28. doi:10.1016/j.jconrel.2004.08.010

[3] W. Xie, P. Xu and Q. Liu, "Antioxidative Activity of Water Soluble Chitosan Derivatives," Bioorganic and $\mathrm{Me}$ dicinal Chemistry Letters, Vol. 11, No. 1, 2001, pp. 16991701. doi:10.1016/S0960-894X(01)00285-2

[4] H. Y. Lin and C. C. Chou, "Antioxidative Activities of Water-Soluble Disaccharide Chitosan Derivatives," Food Research International, Vol. 37, No. 9, 2004, pp. 883-889. doi:10.1016/j.foodres.2004.04.007

[5] K. Esumi, N. Takei and T. Yoshimura, "Antioxidant-Potentiality of Gold-Chitosan Nanocomposites," Colloids and Surfaces B: Biointerfaces, Vol. 32, No. 2, 2003, pp. 117 123. doi:10.1016/S0927-7765(03)00151-6

[6] R. Xing, S. Liu, H. Yu, Q. Zhang, Z. Li and P. Li, "Preparation of Low-Molecular-Weight and High-Sulfate-Content Chitosans under Microwave Radiation and Their Potential Antioxidant Activity in Vitro," Carbohydrate Research, Vol. 339, No. 15, 2004, pp. 2515-2519. doi:10.1016/j.carres.2004.08.013

[7] A. Dávalos, B. Bartolomé and C. Gómez-Cordovés, "Antioxidant Properties of Commercial Grape Juices and Vinegars," Food Chemistry, Vol. 93, No. 2, 2005, pp. 325330. doi:10.1016/j.foodchem.2004.09.030

[8] C. F. Chau, Y. L. Wen and Y. T. Wang, "Effects of the Micronization on the Characteristics and Physicochemical Properties of Insoluble Fibres," Journal of the Science of Food and Agriculture, Vol. 86, No. 14, 2006, pp. 23802386. doi:10.1002/jsfa.2628

[9] K. Shimada, K. Fujikawa, K. Yahara and T. Nakamura, “Antioxidative Properties of Xanthan on the Anti-Oxidation of Soybean Oil in Cyclodextrin Emulsion," Journal of Agricultural and Food Chemistry, Vol. 40, No. 6, 1992, pp. 945-948. doi:10.1021/jf00018a005

[10] G. C. Yen and D. Y. Chung, "Antioxidant Effects of Extracts from Cassia tora L. Prepared under Different Degrees of Roasting on the Oxidative Damage to Biomolecules," Journal of Food Chemistry, Vol. 47, No. 4, 1999, pp. 1326-1332. doi:10.1021/jf9810618
[11] J. Robak and R. J. Gryglewski, "Flavonoids Are Scavengers of Superoxide Anions," Biochemical Pharmacology, Vol. 37, No. 5, 1988, pp. 837-841. doi:10.1016/0006-2952(88)90169-4

[12] R. Pellegrini, N. Proteggente, A. Pannala, A. Yang and C. Rice-Evans, "Antioxidant Activity Applying an Improved ABTS Radical Cation Decolorization Assay," Free Radical Biology \& Medicine, Vol. 26, No. 9-10, 1999, pp. 12311237. doi:10.1016/S0891-5849(98)00315-3

[13] M. J. T. J. Arts, J. S. Dallinga, H.-P. Voss, G. R. M. M. Haenen and A. Bast, "A New Approach to Assess the Total Antioxidant Capacity Using the TEAC Assay," Food Chemistry, Vol. 88, No. 4, 2004, pp. 567-570. doi:10.1016/j.foodchem.2004.02.008

[14] L. C. Wu, H. W. Hsu, Y. C. Chen, C. C. Chiu, Y. I. Lin and J. A. Ho, "Antioxidant and Antiproliferative Activities of Red Pitaya," Food Chemistry, Vol. 95, No. 2, 2006, pp. 319-327. doi:10.1016/j.foodchem.2005.01.002

[15] R. Berg van den, G. R. M. M. Haenen, H. Berg van den and A. Bast, "Applicability of an Improved Trolox Equivalent Antioxidant Capacity (TEAC) Assay for Evaluation of Antioxidant Capacity Measurements of Mixtures," Food Chemistry, Vol. 66, No. 4, 1999, pp. 511-517. doi:10.1016/S0308-8146(99)00089-8

[16] T. Yamaguchi, H. Takamura, T. Matoba and J. Terao, "HPLC Method for Evaluation of the Free Radical-Scavenging Activity of Foods by Using 1,1-Diphenyl-2-picrylhydrazyl. Bioscience," Biotechnology and Biochemistry, Vol. 62, No. 6, 1998, pp. 1201-1204. doi:10.1271/bbb.62.1201

[17] B. Halliwell and S. Chirico, "Lipid Peroxidation: Its Mechanism, Measurement, and Significance," The American Journal of Clinical Nutrition, Vol. 57, No. 5, 1993, pp. 715-725.

[18] P. A. Hyslop, D. B. Hinshaw, W. A. J. Halsey, I. U. Schraufstatter, R. D. Sauerheber, R. G. Spragg, J. H. Jackson and C. G. Cochrane, "Mechanisms of Oxidant-Mediated Cell Injury the Glycolytic and Mitochondrial Pathways of ADP Phosphorylation Are Major Intracellular Targets Inactivated by Hydrogen Peroxide," The Journal of Biological Chemistry, Vol. 263, No. 4, 1988, pp. 1665-1675.

[19] B. Halliwell, M. A. Murcia, S. Chirico and O. I. Aruoma, "Aruoma, Free Radicals and Antioxidants in Food and in Vivo: What They Do and How They Work," Critical Reviews in Food Science and Nutrition, Vol. 35, No. 1-2, 1995, pp. 7-20. doi:10.1080/10408399509527682

[20] P. T. Gardner, T. A. C. White, D. B. McPhail and G. G. Duthie, "The Relative Contribution of Vitamin C, Carotenoids and Phenolics to the Antioxidant Potential of Fruit Juices," Food Chemistry, Vol. 68, No. 4, 2000, pp. 471474. doi:10.1016/S0308-8146(99)00225-3 